\title{
Genome-wide association mapping of flowering time and northern corn leaf blight (Setosphaeria turcica) resistance in a vast commercial maize germplasm set
}

\author{
Delphine Van Inghelandt ${ }^{1,3}$, Albrecht E Melchinger ${ }^{1}$, Jean-Pierre Martinant ${ }^{2}$ and Benjamin Stich ${ }^{1,4^{*}}$
}

\begin{abstract}
Background: Setosphaeria turcica is a fungal pathogen that causes northern corn leaf blight (NCLB) which is a serious foliar disease in maize. In order to unravel the genetic architecture of the resistance against this disease, a vast association mapping panel comprising 1487 European maize inbred lines was used to (i) identify chromosomal regions affecting flowering time (FT) and northern corn leaf blight (NCLB) resistance, (ii) examine the epistatic interactions of the identified chromosomal regions with the genetic background on an individual molecular marker basis, and (iii) dissect the correlation between NCLB resistance and FT.

Results: The single marker analyses performed for 8244 single nucleotide polymorphism (SNP) markers revealed seven, four, and four SNP markers significantly ( $\alpha=0.05$, amplicon wise Bonferroni correction) associated with FT, $\mathrm{NCLB}$, and NCLB resistance corrected for FT, respectively. These markers explained individually between 0.36 and 14.29\% of the genetic variance of the corresponding trait.

Conclusions: The very well interpretable pattern of SNP associations observed for FT suggested that data from applied plant breeding programs can be used to dissect polygenic traits. This in turn indicates that the associations identified for NCLB resistance might be successfully used in marker-assisted selection programs. Furthermore, the associated genes are also of interest for further research concerning the mechanism of resistance to NCLB and plant diseases in general, because some of the associated genes have not been mentioned in this context so far.
\end{abstract}

\section{Background}

Setosphaeria turcica (anamorph Exserohilum turcicum, formerly known as Helminthosporium turcicum) is a fungal pathogen that causes northern corn leaf blight (NCLB) in maize. NCLB is a serious, omnipresent foliar disease $[1,2]$. Infections of maize with NCLB before silking can cause grain yield losses of more than $50 \%$, which are accompanied by a reduction in feed value and the predisposition of infected plants to stalk rot [3].

Plants have evolved qualitative and quantitative resistance to combat pathogens. Qualitative resistance

\footnotetext{
*Correspondence: stich@mpipz.mpg.de

${ }_{1}^{1}$ Institute of Plant Breeding, Seed Science, and Population Genetics, University of Hohenheim, 70593 Stuttgart, Germany

${ }^{4}$ Max Planck Institute for Plant Breeding Research, Carl-von-Linne-Weg 10, 50829 Cologne, Germany

Full list of author information is available at the end of the article
}

typically confers a high level of resistance, is usually race specific, and is based on single, mostly dominantly acting genes ( $\mathrm{R}$ genes; for review see [4]). For NCLB, qualitative resistances have been identified and called $H t$ genes (for Helminthosporium turcicum): Ht1 [5] and HtP [6] were mapped to the long arm of chromosome 2, Ht2 [7] as well as $H t n 1$ [8] were mapped to the long arm of chromosome 8 , and $H t 3$ was the only resistance gene that was ever introgressed from Tripsacum floridanum into maize [9]. These single resistance genes have been backcrossed into a number of widely used inbred lines, where they showed partial dominance and expression dependent on the genetic background [10]. Furthermore, the expression of the $\mathrm{Ht}$ genes is modified by the environment, particularly temperature and light intensity [11]. In addition, qualitative resistances conferred by single genes such as the $H t$ genes tend to be overcome by new, virulent races 
of Setosphaeria turcica e.g. [12,13]. All these aspects limit the practical value of the $H t$ genes and have hampered their use in maize breeding programs.

Quantitative resistances are considered to be oligo- or polygenically inherited and, thus, partially as well as moderatly effective, but race unspecific and durable (for review see [14]). Due to the latter two properties, quantitative resistances are today considered more useful in a breeding context than qualitative resistances. In agrement with this conclusion, the majority of disease resistances deployed in elite varieties of maize are quantitative. However, identification of genes confering quantitative resistance is much more challenging than identifying $R$ genes, owing to their smaller phenotypic effects.

Various studies have been conducted to map quantitative trait loci (QTLs) for resistance to NCLB (for review see [15]). All of them were linkage mapping studies using different types of progenies such as $F_{2}$ or $F_{3}$ generations, $B C_{1}$ generations, or populations of near isogenic lines or recombinant inbred lines. In these studies, QTLs were detected on all maize chromosomes except chromosome ten. Due to the large confidence intervals of QTLs and a restricted allelic sampling in the two parental genotypes, however, the results of linkage mapping studies had so far little impact on resistance breeding. Very recently, NCLB resistance in maize was dissected using the nested asociation mapping (NAM) population [16], which offers the advantage of a higher mapping resolution and a broader allelic sampling than the above mentioned linkage mapping studies. Nevertheless, population-based association mapping has the potential of resulting in an even higher mapping resolution and broader allelic sampling compared to NAM [17]. To our knowledge, however, no genome-wide population-based association mapping study has been yet conducted for NCLB resistance in maize.

Resistance genes identified by linkage or association mapping might affect the disease either directly or indirectly $(c f .[18,19])$. Genes affecting plant growth and development or time to flowering (FT) fall in the latter class. Especially for diseases caused by necrotrophic pathogens such as Setosphaeria turcica, which are more severe on senescing leaf tissue after anthesis, a relationship betweeen plant disease resistance and FT might be expected [20]. Despite the contradictory results from earlier phenotypic analyses (e.g. [21,22]), some QTLs for NCLB resistance found in meta-analyses colocalized with those for FT and maturity (for review see [15]). However, in contrast to these linkage mapping studies, our association analysis will allow to discriminate with a high mapping resolution between pleiotropy and linkage of QTL for NCLB resistance and FT (cf. [23]).

In this study, a large association mapping panel comprising 1487 elite maize inbred lines was used to (i) identify
Table 1 First and second-degree statistics for flowering time (FT) and northern corn leaf blight (NCLB) resistance of the 1487 phenotyped and genotyped inbred lines

\begin{tabular}{lcc}
\hline & \multicolumn{2}{c}{ Trait } \\
\cline { 2 - 3 } Parameter & FT & NCLB \\
\hline mean $\left(M_{i}\right)$ & 55.2 & 4.8 \\
range $\left(M_{i}\right)$ & $34.0-73.5$ & $0.5-10.0$ \\
$\sigma_{G}^{2}$ & 42.34 & 3.24 \\
$\sigma_{G . E}^{2}$ & 2.21 & 0.63 \\
$h^{2}$ & 0.95 & 0.87 \\
\hline
\end{tabular}

FT is measured in number of days to female flowering after June 1. NCLB is rated from 1-9 (sensitive-resistant).

$M_{i}$ is the adjusted entry mean of genotype $i$ calculated across all environments. $\sigma_{G}^{2}$ and $\sigma_{G . E}^{2}$ are the genotypic and genotype $\times$ environment interaction variances.

$h^{2}$ is the heritability on entry an mean basis.

chromosomal regions affecting FT and NCLB resistance, (ii) examine the epistatic interactions of the identified chromosomal regions with the genetic background on an individual molecular marker basis, and (iii) dissect the correlation between NCLB resistance and FT.

\section{Results}

For the whole set of phenotyped and genotyped inbred lines, the heritability of FT and NCLB resistance was 0.95 and 0.85 , respectively (Table 1 ). NCLB was significantly $(r=0.53, \alpha=0.05)$ correlated with FT (Figure 1) in the whole set of 1487 inbred lines. The Pearson correlation coefficient was lower within the four heterotic pools and ranged from 0.27 (Stiff Stalk; SSS) to 0.33 (Flint) (Figure 1).

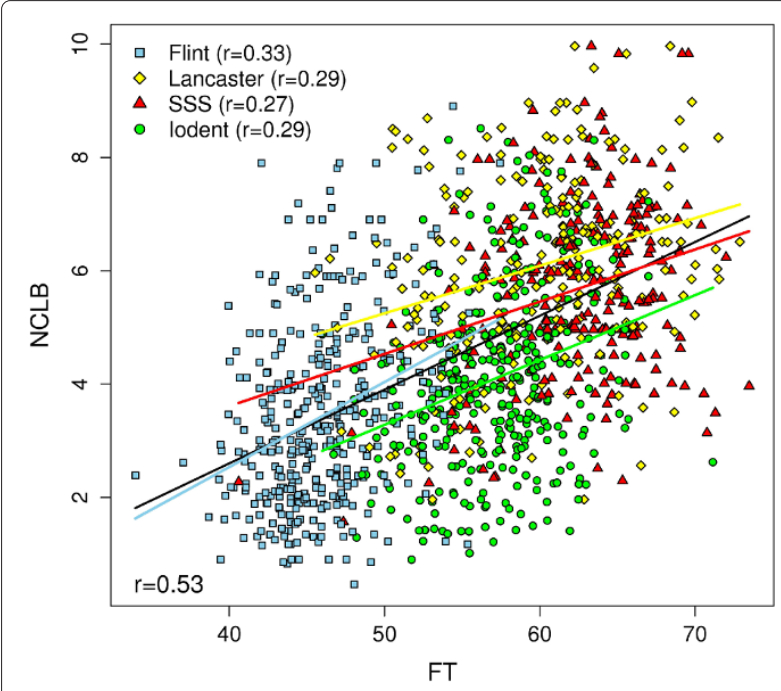

Figure 1 Regression curves of the adjusted entry means of flowering time (FT) vs. northern corn leaf blight resistance (NCLB) for the entire set of 1487 maize inbred lines as well as the individual heterotic pools. $r$ is Pearson's correlation coefficient between the two traits. 


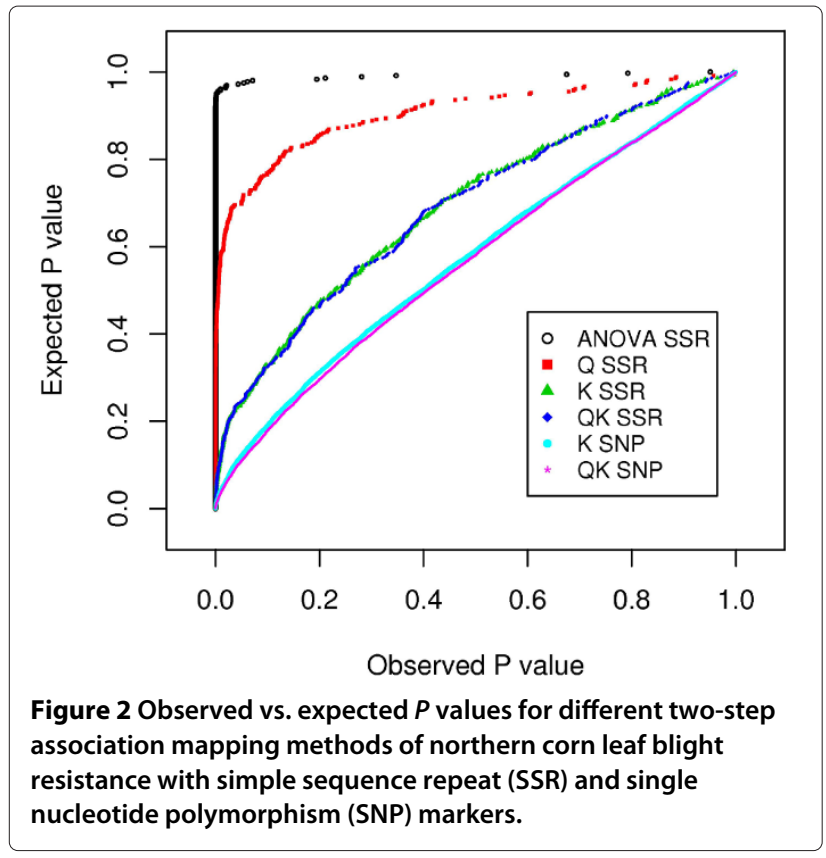

For the SSR markers, the observed $P$ values obtained with the QK and $\mathrm{K}$ model showed in comparison to the ANOVA and the Q model a smaller deviation from the uniform distribution (Figure 2). Furthermore, the mean squared difference (MSD) between observed and expected $P$ values was slightly smaller for the QK model than for the $\mathrm{K}$ model. In addition to the SSRs, this was also true for the SNP markers $(0.041$ versus $0.042 ; 0.005$ versus 0.007 , respectively). The population background structure accounted for 21,6 , and $2 \%$ of the genetic variation in FT, NCLB and NCLB resistance corrected for FT $\left(\operatorname{NCLB}_{F T}\right)$, respectively.

In single marker analyses, seven, four, and four SNP markers were significantly $(\alpha=0.05$, amplicon wise Bonferroni correction) associated with FT, NCLB, and $\mathrm{NCLB}_{F T}$ resistance, respectively (Figure 3). For FT, the seven SNPs explained individually between 5.39 and $14.29 \%$ of the genetic variance, whereas all SNPs together explained $13.20 \%$ (Table 2). For NCLB and NCLB $F T$, the four SNPs explained between 3.32 to $4.78 \%$ and between 0.36 to $6.76 \%$ of the genetic variance, respectively. In a simultaneous fit, they explained 8.18 and $9.48 \%$ of the genetic variance of $\mathrm{NCLB}$ and $\mathrm{NCLB}_{F T}$, respectively.

In the Flint, Lancaster, SSS, and Iodent pool, two, four, two, and six SNPs were significantly $(\alpha=0.05$, amplicon wise Bonferroni correction) associated with FT (Additional file 1: Figure S1), which explained in a simultaneous fit $1.87,22.99,21.35$, and $25.50 \%$ of the genetic variance in the corresponding heterotic pools (Table 3). For NCLB, two and six significantly associated SNP markers were identified in the SSS and Iodent pool, respectively, but none for the Flint and Lancaster pools
(Additional file 2: Figure S2). Similarly, one and three SNPs were found to be significantly associated with $\mathrm{NCLB}_{F T}$ in the SSS and Iodent pool, respectively (Additional file 3: Figure S3). The SNPs associated with NCLB explained in a simultaneous fit 9.38 and $28.94 \%$ of the genetic variance, whereas those associated with $\mathrm{NCLB}_{F T}$ explained 0 and $23.20 \%$ of the genetic variance in the SSS and Iodent pool, respectively (Table 3 ).

The three rounds of multiple forward regression revealed for the whole set of 1487 inbred lines three SNP markers to be significantly associated with FT and NCLB, but only two with $\operatorname{NCLB}_{F T}$ (Table 4). The simultaneous fit of these SNPs explained 16.65, 7.62, and $6.13 \%$ of the genetic variance of $\mathrm{FT}, \mathrm{NCLB}$, and $\mathrm{NCLB}_{F T}$, respectively. Significant $(\alpha=0.05$, amplicon wise Bonferroni correction) epistatic interactions were identified between the significant SNPs from the single marker analyses as well as the multiple forward regression procedure and all other SNPs for FT and NCLB resistance, respectively (Figure 4). No significant epistatic interactions were detected for $\operatorname{NCLB}_{F T}$. The epistatic interactions found for the two traits explained a maximum of $5 \%$ of the genetic variance (Additional file 4: Figure S4).

\section{Discusssion}

Statistical aspects of association analysis

\section{One-step vs. Two-step approaches}

In all genetic mapping experiments, the one-step approach, in which phenotypic and genotypic data are analysed in a single step, is the only fully efficient analysis [24]. However, a comparison with the two-step analysis showed only a marginal increase in the empirical type I error rate [25]. As the two-step analysis is computationally much less demanding, we used this approach in view of the large data set analysed in our study.

\section{Alternative association mapping models}

Several methods for association analysis in plants have been described recently [25-27]. In order to identify the most appropriate association mapping method for our data set, we compared for background SSR markers several models with respect to the deviation of the $P$ values from a uniform distribution [28]. This is because under the assumption that our SSR markers are unlinked to functional polymorphism due to their low genome coverage [29], it is expected that the $P$ values observed for an association mapping approach are uniformly distributed (cf. [26]). The mean of squared difference (MSD) between observed and expected $P$ values of all marker loci was therefore calculated as a measure for the deviation of the $P$ values from a uniform distribution. The results of these analyses (Figure 2) suggested that the QK method [26] with kinship matrix $\mathbf{K}$ calculated as the fraction of shared alleles [27] was the most appropriate method for our data set with respect to the adherence to the nominal $\alpha$ level. 


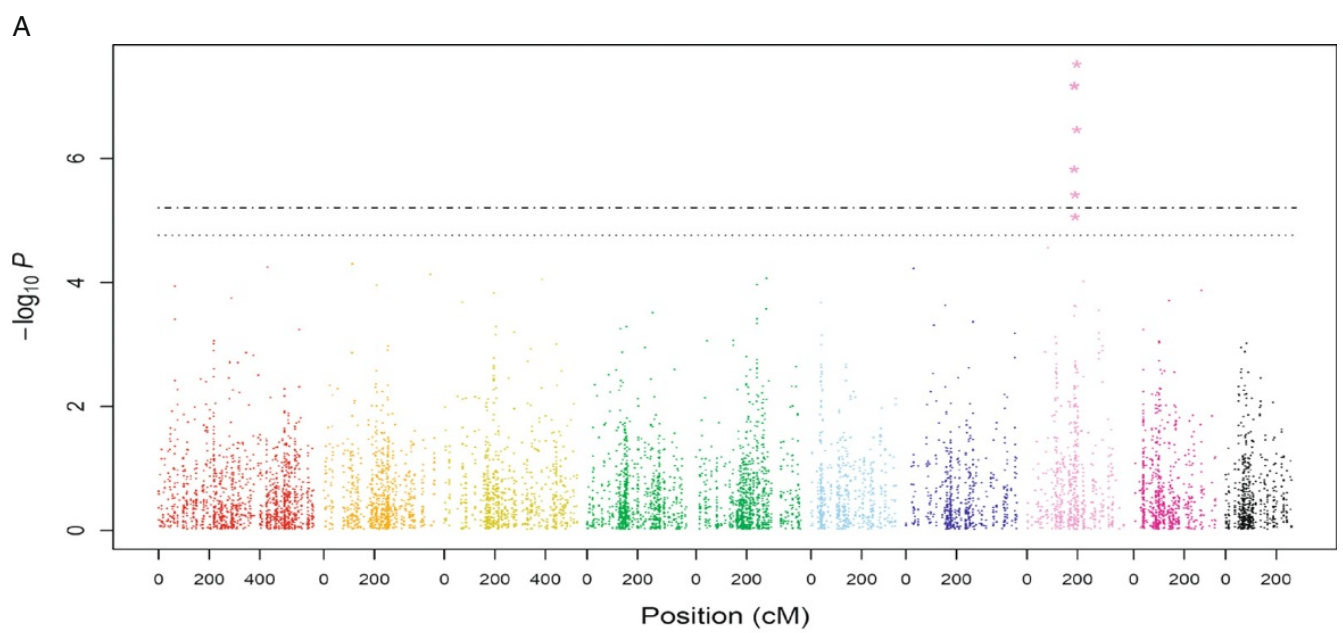

B

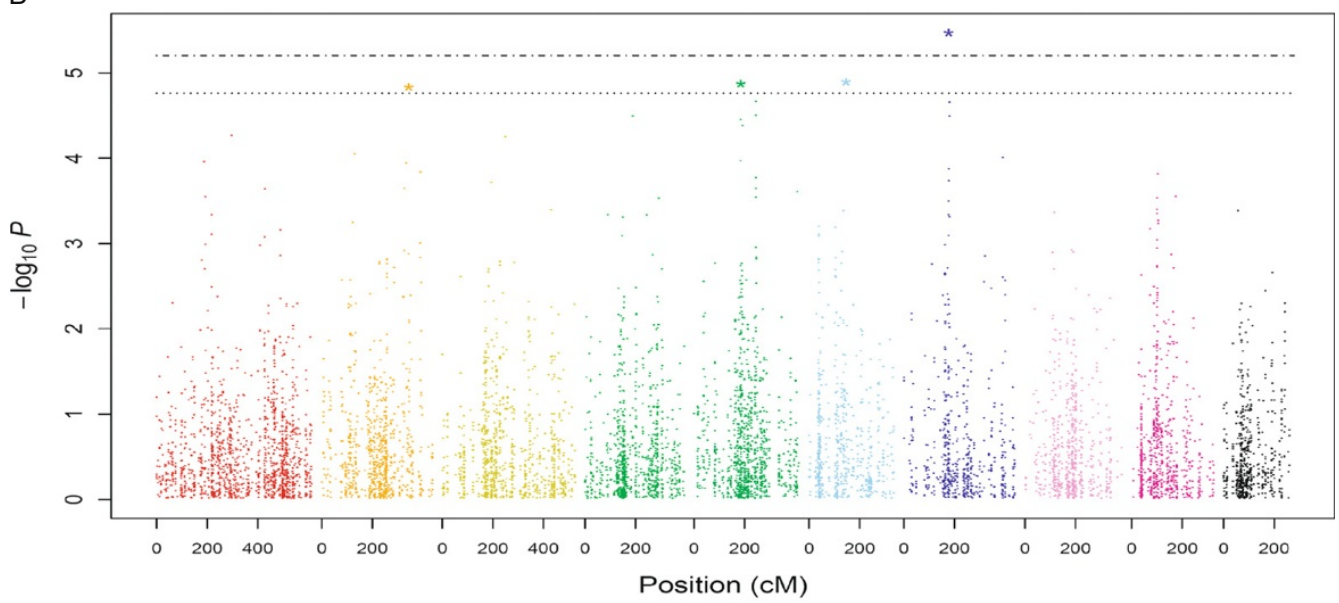

C

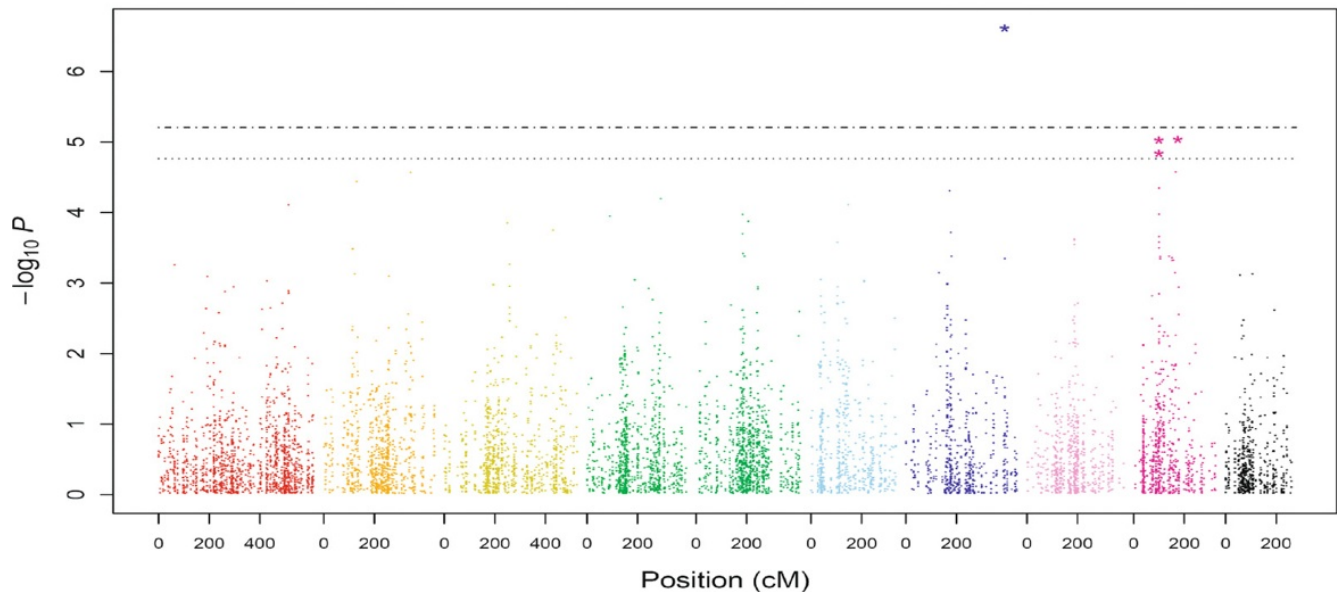

Figure 3 Genome-wide $P$ values for association analysis of flowering time (FT; A), northern corn leaf blight (NCLB; B), and FT corrected NCLB resistance $(C)$ for the entire set of $\mathbf{1 4 8 7}$ maize inbred lines. The ten colors represent the ten chromosomes. The horizontal, doted and dashed-doted lines correspond to a nominal 5\% significance threshold with Bonferroni and amplicon-wise Bonferroni correction, respectively. Significant $P$ values are represented by a star. 
Table 2 Single nucleotide polymorphism (SNP) marker loci significantly associated with flowering time (FT), northern corn leaf blight (NCLB), and FT corrected NCLB (NCLB $F$ ) resistance for the entire set of 1487 maize inbred lines as identified by single marker analysis

\begin{tabular}{|c|c|c|c|c|c|c|c|c|c|}
\hline Trait & $\begin{array}{c}\text { Marker } \\
\text { locus }\end{array}$ & Gene & $\begin{array}{l}\text { Chr. } \\
\text { bin }\end{array}$ & $\begin{array}{l}\text { Position } \\
\text { (cM) }\end{array}$ & $\begin{array}{l}\text { Position } \\
\text { (bp) }\end{array}$ & $P$ value & $\begin{array}{c}\text { Allele } \\
1 / 2\end{array}$ & $\begin{array}{c}\text { Effect } \\
\text { Allele 1-2 }\end{array}$ & $\begin{array}{l}p_{G} \\
(\%)\end{array}$ \\
\hline \multirow[t]{8}{*}{$\overline{F T}$} & M_00048149 & LE00126 & 8.05 & 187.40 & 128429853 & $1.5 e-06^{* *}$ & $\mathrm{G} / \mathrm{A}$ & 1.66 & 10.72 \\
\hline & M_00041827 & AY104033 & 8.05 & 188.17 & 130730508 & $6.8 \mathrm{e}-08^{* *}$ & $\mathrm{C} / \mathrm{T}$ & 2.21 & 9.75 \\
\hline & M_00041828 & AY104033 & 8.05 & 188.17 & 130730581 & $6.6 \mathrm{e}-08^{* *}$ & $\mathrm{C} / \mathrm{T}$ & 2.21 & 9.81 \\
\hline & M_00044984 & CL4016 & 8.05 & 191.34 & 131678990 & $3.8 \mathrm{e}-06^{* *}$ & $\mathrm{~T} / \mathrm{C}$ & 1.49 & 6.34 \\
\hline & M_00044985 & CL4016 & 8.05 & 191.34 & 131678953 & $8.7 e-06^{*}$ & $A / G$ & 1.43 & 5.39 \\
\hline & M_00049486 & LE00214 & 8.05 & 197.92 & 145084250 & $3.0 \mathrm{e}-08^{* *}$ & $\mathrm{C} / \mathrm{T}$ & -2.03 & 14.29 \\
\hline & M_00049487 & LE00214 & 8.05 & 197.92 & 145084209 & $3.4 \mathrm{e}-07^{* *}$ & $C / A$ & -1.66 & 9.10 \\
\hline & Simultaneous fit & & & & & & & & 13.20 \\
\hline \multirow[t]{4}{*}{ NCLB } & M_00040077 & AY105483 & 2.08 & 343.92 & 217079800 & $1.4 \mathrm{e}-05^{*}$ & G/A & -0.54 & 3.32 \\
\hline & M_00043741 & AY111579 & 6.05 & 146.44 & 145332550 & $1.3 e-05^{*}$ & $\mathrm{C} / \mathrm{T}$ & 0.61 & 4.27 \\
\hline & M_00048400 & LE00018 & 7.02 & 178.00 & 100239763 & $3.3 e-06^{* *}$ & $\mathrm{~T} / \mathrm{C}$ & -0.47 & 4.78 \\
\hline & Simultaneous fit & & & & & & & & 8.18 \\
\hline \multirow[t]{5}{*}{$\mathrm{NCLB}_{F T}$} & M_00045254 & AY107778 & 7.04 & 391.46 & 169937062 & $2.4 \mathrm{e}-07^{* *}$ & $\mathrm{~T} / \mathrm{C}$ & -1.89 & 0.36 \\
\hline & M_00041355 & AY107035 & 9.03 & 99.76 & 43679500 & $1.4 \mathrm{e}-05^{*}$ & $\mathrm{~A} / \mathrm{C}$ & -0.58 & 6.76 \\
\hline & M_00041356 & AY107035 & 9.03 & 99.76 & 43679530 & $9.3 e-06^{*}$ & $\mathrm{G} / \mathrm{A}$ & -0.58 & 6.66 \\
\hline & M_00039528 & AY104217 & 9.05 & 174.09 & 135147555 & $9.1 e-06^{*}$ & $A / G$ & -0.72 & 4.04 \\
\hline & Simultaneous fit & & & & & & & & 9.48 \\
\hline
\end{tabular}

$p_{G}$ is the proportion of the explained genotypic variance.

${ }^{*}$ Significant at $P<0.05$ with amplicon-wise Bonferroni correction.

${ }^{* *}$ Significant at $P<0.05$ with Bonferroni correction.

The use of the QK method for the SNP-phenotype association analysis, however, resulted in fewer associations compared to the K method (data not shown). Because it is not possible to determine whether these associations were lost due to the lower power of the QK method or as they are caused by population structure, we decided for the conservative way and discussed below only the results of the QK method.

An alternative to single marker analysis is haplotypebased association analysis. This requires the building of haplotypes based on the extent of LD between the single markers. In the germplasm examined in our study, the average extent of LD between SNPs within amplicons varied from $r^{2}=0.253$ to $r^{2}=0.304$, depending on the heterotic pools investigated [29]. In the case of such relatively low levels of LD, the number of haplotypes per amplicon is high and therefore their frequencies low. This in turn leads to a low power for detecting associations by a haplotype-based analysis. Therefore, we think haplotypebased association mapping is no promising strategy in the case of our study.

\section{Corrections for multiple testing}

In genome-wide association mapping studies with $n$ molecular markers, the same statistical test is performed $n$ times at the significance level $\alpha$. Across all tests, however, the experimental type I error rate will be much higher than $\alpha$ (e.g. [30]). To overcome this problem and obtain an appropriate significance threshold, it was recommended to apply the Bonferroni correction [31], where the $\alpha$ level is divided by the number of independent tests. However, determining the number of independent tests is not straight forward in the context of genome-wide association mapping studies. Owing to the correlation structure among markers, it would be overly conservative to use the total number of markers as a substitute for the number of independent tests [32]. As the 8 244 SNP markers of our study were derived from 2973 amplicons and SNPs from the same amplicon tend to show higher correlations than SNPs from different amplicons [29], we used besides the total number of SNPs also the number of amplicons as correction factor for the Bonferroni procedure.

\section{Single marker analysis vs. multiple forward regression}

An efficient approach to identify significant markerphenotype associations inspite of the collinearity between markers might be multiple forward regression (cf. [33]). We applied this approach in the context of mixed-model analyses and detected SNPs that have not been detected with the single marker analysis (Table 4). Furthermore, 
Table 3 Single nucleotide polymorphism (SNP) marker loci significantly associated with flowering time (FT), northern corn leaf blight (NCLB), and FT corrected NCLB $\left(N_{C L B}\right)$ resistance in the different heterotic pools as identified by single marker analysis. $p_{G}$ is the proportion of the explained genotypic variance and SSS is the Stiff Stalk heterotic pool

\begin{tabular}{|c|c|c|c|c|c|c|c|c|c|c|}
\hline Trait & Pool & $\begin{array}{c}\text { Marker } \\
\text { locus }\end{array}$ & Gene & $\begin{array}{l}\text { Chr. } \\
\text { bin }\end{array}$ & $\begin{array}{c}\text { Position } \\
\text { (cM) }\end{array}$ & $\begin{array}{c}\text { Position } \\
\text { (bp) }\end{array}$ & $P$ value & $\begin{array}{c}\text { Allele } \\
1 / 2\end{array}$ & $\begin{array}{c}\text { Effect } \\
\text { Allele 1-2 }\end{array}$ & $\begin{array}{r}p_{G} \\
(\%)\end{array}$ \\
\hline \multirow[t]{18}{*}{$\overline{\mathrm{FT}}$} & Flint & M_00049486 & LE00214 & 8.05 & 197.92 & 145084250 & $4.3 e-06^{* *}$ & $C / T$ & -2.73 & 1.52 \\
\hline & & M_00047185 & LE00173 & 9.02 & 75.54 & 18327094 & $5.2 \mathrm{e}-06^{* *}$ & $A / T$ & -3.61 & 0.00 \\
\hline & & Simultaneous fit & & & & & & & & 1.87 \\
\hline & Lancaster & M_00045062 & MAGI39472 & 3.07 & 346.79 & 195315842 & $1.5 \mathrm{e}-05^{*}$ & $\mathrm{C} / \mathrm{T}$ & 2.83 & 7.48 \\
\hline & & M_00045063 & MAGI39472 & 3.07 & 346.79 & 195315842 & $1.5 \mathrm{e}-05^{*}$ & $T / G$ & 2.83 & 7.48 \\
\hline & & M_00048149 & LE00126 & 8.05 & 187.40 & 128429853 & $5.8 \mathrm{e}-06^{* *}$ & $\mathrm{G} / \mathrm{A}$ & 3.15 & 10.20 \\
\hline & & M_00049487 & LE00214 & 8.05 & 197.92 & 145084209 & $1.9 \mathrm{e}-06^{* *}$ & $C / A$ & -4.20 & 13.64 \\
\hline & & Simultaneous fit & & & & & & & & 22.99 \\
\hline & SSS & M_00048750 & LE00097 & 2.02 & 95.07 & 5820265 & $2.4 \mathrm{e}-06^{* *}$ & $\mathrm{G} / \mathrm{A}$ & 13.62 & 14.80 \\
\hline & & M_00047756 & LE00008 & 5.03 & 186.65 & 67510540 & $5.6 e-06^{* *}$ & $C / T$ & -6.25 & 16.76 \\
\hline & & Simultaneous fit & & & & & & & & 21.35 \\
\hline & lodent & M_00039634 & AY106491 & 8.03 & 115.95 & 65244298 & $1.7 e-05^{*}$ & $\mathrm{G} / \mathrm{A}$ & 3.57 & 18.18 \\
\hline & & M_00044984 & CL4016 & 8.05 & 191.34 & 131678990 & $1.1 \mathrm{e}-05^{*}$ & $\mathrm{~T} / \mathrm{C}$ & 1.95 & 14.82 \\
\hline & & M_00044985 & CL4016 & 8.05 & 191.34 & 131678953 & $1.2 \mathrm{e}-05^{*}$ & $A / G$ & 1.98 & 15.18 \\
\hline & & M_00040388 & AY106357 & 8.05 & 192.10 & 134066305 & $1.2 \mathrm{e}-06^{* *}$ & $\mathrm{~T} / \mathrm{C}$ & 2.51 & 17.14 \\
\hline & & M_00042146 & AY109558 & 8.05 & 192.10 & 135061056 & $1.3 e-05^{*}$ & $\mathrm{~T} / \mathrm{C}$ & 2.30 & 18.77 \\
\hline & & M_00046254 & HDT102 & 8.05 & 192.26 & 136131540 & $1.4 \mathrm{e}-05^{*}$ & $\mathrm{~T} / \mathrm{A}$ & -2.16 & 17.55 \\
\hline & & Simultaneous fit & & & & & & & & 25.50 \\
\hline
\end{tabular}


Table 3 Single nucleotide polymorphism (SNP) marker loci significantly associated with flowering time (FT), northern corn leaf blight (NCLB), and FT corrected NCLB $\left(N C L B_{F T}\right)$ resistance in the different heterotic pools as identified by single marker analysis. $p_{G}$ is the proportion of the explained genotypic variance and SSS is the Stiff Stalk heterotic pool (Continued)

\begin{tabular}{|c|c|c|c|c|c|c|c|c|c|c|}
\hline \multirow[t]{10}{*}{$\overline{N C L B}$} & SSS & M_00046267 & HAM101 & 2.08 & 322.28 & 212539314 & $1.2 \mathrm{e}-06^{* *}$ & $\mathrm{C} / \mathrm{T}$ & 1.09 & 9.38 \\
\hline & & M_00046268 & HAM101 & 2.08 & 322.28 & 212539211 & $7.3 e-07^{* *}$ & $\mathrm{~T} / \mathrm{C}$ & 1.10 & 11.30 \\
\hline & & Simultaneous fit & & & & & & & & 9.38 \\
\hline & lodent & M_00048759 & LE00099 & 3.05 & 227.44 & 160670670 & $1.4 \mathrm{e}-05^{*}$ & $\mathrm{~A} / \mathrm{G}$ & 1.78 & 6.05 \\
\hline & & M_00044733 & AZM452718 & 5.03 & 187.81 & 69119810 & $5 e-06^{* *}$ & $\mathrm{G} / \mathrm{A}$ & -0.90 & 8.49 \\
\hline & & M_00046712 & AY103770 & 9.03 & 111.71 & 99416354 & $6 e-06^{* *}$ & $\mathrm{G} / \mathrm{C}$ & 1.25 & 10.33 \\
\hline & & M_00046713 & AY103770 & 9.03 & 111.71 & 99416416 & $9.7 e-07^{* *}$ & $\mathrm{~T} / \mathrm{G}$ & -1.32 & 11.49 \\
\hline & & M_00040630 & AY105678 & 9.05 & 151.55 & 130918789 & $9.1 \mathrm{e}-06^{*}$ & $\mathrm{C} / \mathrm{T}$ & -0.81 & 10.02 \\
\hline & & M_00040631 & AY105678 & 9.05 & 151.55 & 130918887 & $1.1 \mathrm{e}-05^{*}$ & $\mathrm{~T} / \mathrm{C}$ & -0.79 & 9.08 \\
\hline & & Simultaneous fit & & & & & & & & 28.94 \\
\hline \multirow[t]{6}{*}{$\mathrm{NCLB}_{F T}$} & SSS & M_00046331 & HAM101 & 2.08 & 322.10 & 212537417 & $5.9 e-06^{* *}$ & $\mathrm{~A} / \mathrm{T}$ & -1.01 & 0.00 \\
\hline & & Simultaneous fit & & & & & & & & 0.00 \\
\hline & lodent & M_00040577 & AY105760 & 9.03 & 99.89 & 50762538 & $1.3 e-05^{*}$ & $\mathrm{~T} / \mathrm{G}$ & 0.90 & 12.87 \\
\hline & & M_00040630 & AY105678 & 9.05 & 151.55 & 130918789 & $5 e-07^{* *}$ & $\mathrm{C} / \mathrm{T}$ & -0.97 & 18.89 \\
\hline & & M_00040631 & AY105678 & 9.05 & 151.55 & 130918887 & $5.3 e-07^{* *}$ & $\mathrm{~T} / \mathrm{C}$ & -0.96 & 15.99 \\
\hline & & Simultaneous fit & & & & & & & & 23.20 \\
\hline
\end{tabular}

"Significant at $P<0.05$ with amplicon-wise Bonferroni correction.

** Significant at $P<0.05$ with Bonferroni correction. 
Table 4 Simultaneous fit of single nucleotide polymorphism (SNP) markers identified by three rounds of multiple forward regression to be significantly ( $\alpha=0.05$, amplicon wise Bonferroni correction) associated with flowering time (FT), northern corn leaf blight (NCLB), and FT corrected NCLB (NCLB $F$ ) resistance for the entire set of 1487 maize inbred lines. $p_{G}$ is the proportion of the explained genotypic variance

\begin{tabular}{|c|c|c|c|c|c|c|c|c|}
\hline Trait & $\begin{array}{c}\text { Marker } \\
\text { locus }\end{array}$ & Gene & $\begin{array}{l}\text { Chr. } \\
\text { bin }\end{array}$ & $\begin{array}{c}\text { Position } \\
\text { (cM) }\end{array}$ & $\begin{array}{c}\text { Position } \\
\text { (bp) }\end{array}$ & $\begin{array}{c}\text { Allele } \\
1 / 2\end{array}$ & $\begin{array}{c}\text { Effect } \\
\text { Allele 1-2 }\end{array}$ & $\begin{array}{l}p_{G} \\
(\%)\end{array}$ \\
\hline \multirow[t]{4}{*}{ FT } & M_00049486 & LE00214 & 8.05 & 197.92 & 145084250 & $\mathrm{C} / \mathrm{T}$ & -1.99 & \\
\hline & M_00041828 & AY104033 & 8.05 & 188.17 & 130730581 & $C / T$ & 1.93 & \\
\hline & M_00044069 & MAGI31942 & 1.07 & 430.42 & 207180270 & $\mathrm{G} / \mathrm{A}$ & 2.87 & \\
\hline & & & & & & & & 16.65 \\
\hline \multirow[t]{4}{*}{ NCLB } & M_00048400 & LE00018 & 7.02 & 178.00 & 100239763 & $\mathrm{~T} / \mathrm{C}$ & -0.49 & \\
\hline & M_00041320 & AY112216 & 5.03 & 185.02 & 67600206 & $C / T$ & -0.51 & \\
\hline & M_00047997 & LE00080 & 5.05 & 245.04 & 181521588 & $\mathrm{~A} / \mathrm{C}$ & 0.63 & \\
\hline & & & & & & & & 7.62 \\
\hline \multirow[t]{3}{*}{$\mathrm{NCLB}_{F T}$} & M_00045254 & AY107778 & 7.04 & 391.46 & 169937062 & $\mathrm{~T} / \mathrm{C}$ & -1.91 & \\
\hline & M_00039528 & AY104217 & 9.05 & 174.09 & 135147555 & $A / G$ & -0.73 & \\
\hline & & & & & & & & 6.13 \\
\hline
\end{tabular}

the three SNPs identified with the former method for FT explained a higher proportion of the genetic variance than those identified by using the latter method. These results corroborate the appropriateness of multiple forward regression procedures for association analyses.

For NCLB and NCLB ${ }_{F T}$, the SNPs identified by this approach, however, explained in a simultaneous fit a lower proportion of the genetic variance than the SNPs identified by the single marker analysis (Table 2; 4). This finding might be explained by the significance levels applied during the single marker analysis, which are not directly comparable to those of the multiple forward regression. Furthermore, since multiple forward regression for mixed-model approaches is computationally demanding, we were able to perform only three selection steps resulting in a maximum of three selected SNP markers and this provides another explanation for our findings. Therefore, in order to take full advantage of multiple forward regression, more efficient computation algorithms are required.

\section{Identified SNP-phenotype associations}

In the entire germplasm set, the population structure explained $21 \%$ of the genetic variation of FT. This finding suggested that sufficient genetic variation remains for detection of SNP-FT associations. For FT, we observed for the single marker analysis a strong $P$ value peak on bin 8.05, which comprised seven SNPs from four genes (Figure 3). Furthermore, this region was identifed by the multiple forward regression approach (Table 4). Earlier studies recognized this chromosomal region as a hot spot for FT QTLs and genes $([34,35]$ and references cited in there). The physical map positions of the significantly associated SNPs ranged from 128429853 to 145084250 bp. The observed $P$ value peak at about $130 \mathrm{Mbp}$ is in proximity to Vgt1, a non-coding sequence regulating the flowering time gene ZmRap2.7 [36]. However, the close consideration of that region revealed an additional $P$ value peak at about $145 \mathrm{Mbp}$ (Additional file 5: Figure S5). This observation might suggest that in addition to Vgt1 a second gene could be involved in FT control in this region. However, the region identified in our study does not correspond to Vgt2 [37], as the latter FT QTL has been mapped to the other side of Vgt1 towards the top of the chromosome. Since the average linkage disequilibrium (LD) among the significantly associated SNPs in this region was high, these SNPs are not necessarily located in the causal genes, but the association might be due to SNPs in strong LD with polymorphisms in the causal genes (cf. [38]). This, however, requires further research.

Another gene that is frequently proposed to contribute to variation of FT in maize is Dwarf8 (D8) (e.g. [39]). Even though our study included six SNPs from $D 8$, we did not find any significant association in bin 1.10 where $D 8$ is located. Our observation is in accordance with the results of [40], who observed no significant association for $D 8$ in a set of European maize inbred lines. These findings might be explained by a correlation of the allele frequencies of polymorphisms in $D 8$ with population structure in the examined germplasm. When correcting for population structure, it will be impossible to identify such polymorphisms in association analyses [40].

In addition to the SNPs from the Vgt1 region, we identified based on the multiple forward regression approach a SNP from bin 1.07 to be significantly associated with FT (Table 4). This SNP might be located in the QTL (near SSR umc1833) upstream of $D 8$ detected in a meta-analysis 


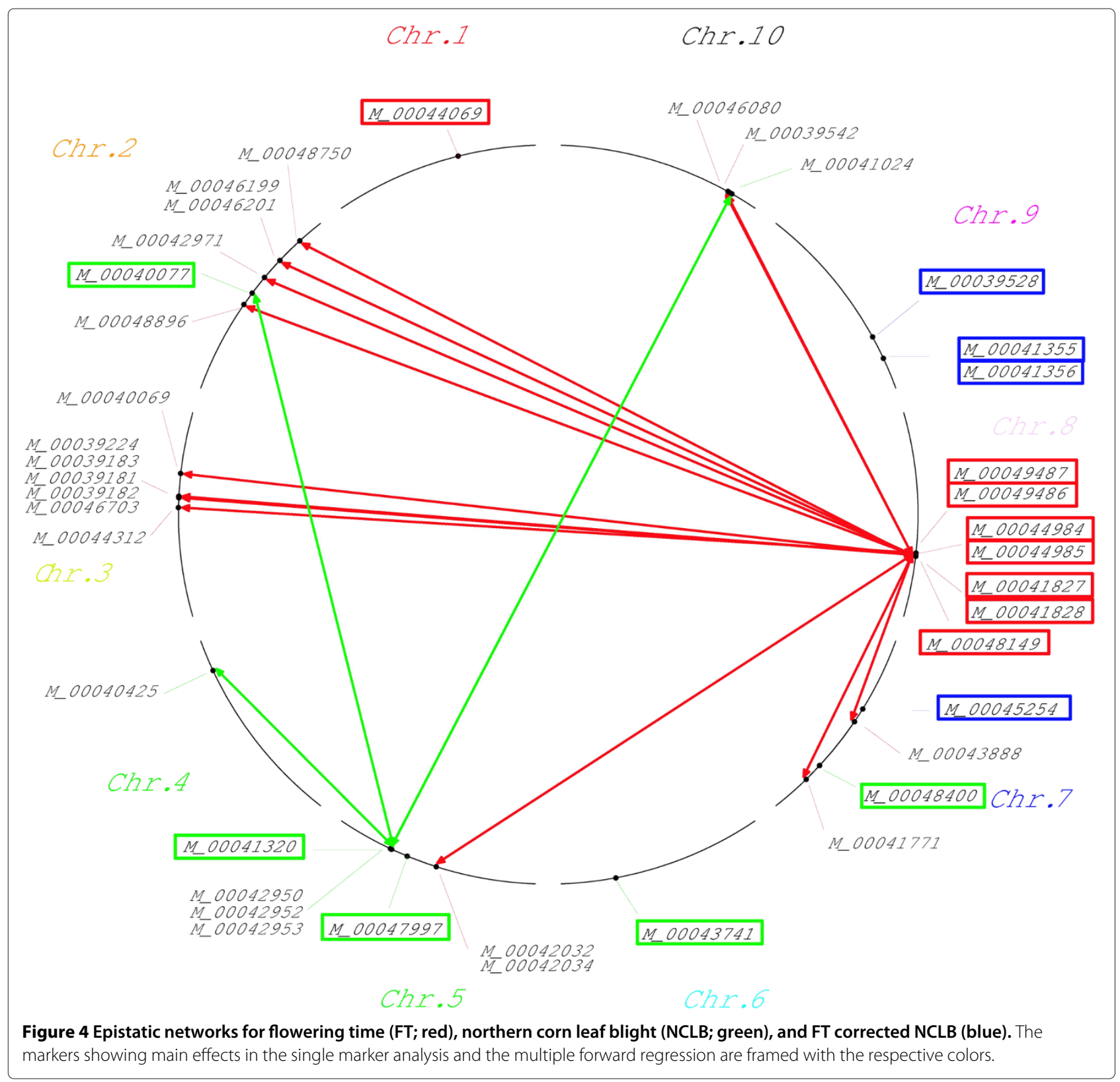

[34] and appears in a P-type R2R3 Myb transcription factor. Since various transcription factors such as $L H Y$ [41] or CCA1 [42] are known to regulate FT in model species, our finding might suggest that this gene is functionally involved in FT regulation of maize.

In conclusion, we observed for FT in maize a very well interpretable pattern of SNP associations that is in harmony with previous genetic analyses. This illustrates that data from practical plant breeding programs can be used not only to dissect oligogenic [43] but also polygenic traits. Furthermore, our findings suggest that the SNPNCLB associations described below might be successfully used in marker-assisted selection programs. We identified five genome regions (four from single marker analyses, one from multiple forward regression) to be significantly associated with NCLB resistance (Table 2; 4) which is considerably lower than the number of genome regions identified by [16]. This finding is most probably due to the different significance thresholds and study designs used.

None of the associations found in our studies was located in bin 8.05, where earlier studies mapped the qualitative NCLB resistance genes $H t 2$ and $H t n 1[7,8]$. Both these genes have been identified in exotic germplasm (Australia, Mexico) and, thus, the resistance alleles might be absent in European elite germplasm. Furthermore, converted inbred lines carrying these introgressed qualitative 
resistance genes were not included in our study in order to prevent any complications with the identification of quantitative resistance genes.

One SNP identified to be significantly associated with NCLB was located in bin 2.08 where the qualitative resistance genes $H t 1$ and $H t P$ have been identified $[5,6]$ and where a QTL was found by [16]. The physical map positions of $H t 1$ and this SNP, however, differ by about 10 Mbp. Nevertheless, the SNP is located within the interval made up by the two closest flanking markers of HtP [6]. Whether this gene, coding for a nonspecific lipid-transfer protein 3 precursor, contributes directly to NCLB resistance or is in LD with the causal gene warrants further research. The same was true for the SNP located in a gene of unknown function in bin 6.05, which resides within the confidence interval of a QTL affecting the incubation period (IP) of NCLB in maize [44] and was located close by a QTL affecting NCLB resistance and IP [16].

Three SNPs significantly associated with NCLB resistance were located in bins 5.03, 5.05, and 7.02 (Table 2; 4) and in each case, a distinct peak of $P$ values was observed (Figure 3). Since all regions have been previously reported to contribute to variation in NCLB resistance $[15,45,46]$, this finding suggests that the identified SNPs are either located in or closely linked with the causal genes (cf. [47]). In contrast to the SNP in bin 5.03, which is located in a gene of unknown function, the SNP in bin 5.05 is located in GPC4, a member of the glyceraldehyde-3-phosphate dehydrogenase gene family, which is involved in sugar metabolism and shows expression differences upon anaerobiosis as well as heat shock [48]. [16] found also a QTL in this region for which a candidate gene was an aldehyde dehydrogenase. The SNP in bin 7.02 is located in a DBF1 like gene, which is a member of the Apetala 2/Ethylene transcription factor family [49] and supposed to have a function in abiotic stress responses and especially dessication tolerance $[49,50]$.

\section{Dissecting the correlation between FT and NCLB}

The results of our study indicated that FT and NCLB resistance are correlated across all heterotic pools $(r=$ 0.53 , Figure 1). This correlation can be explained by the fact that NCLB is a necrophytic disease and, thus, tends to progress more rapidly on senescing tissues [20]. However, the correlations in the individual heterotic pools were only moderate (Flint: 0.33, Lancaster: 0.29, SSS: 0.27 , and SSS: 0.29 ). This suggests that the overall correlation relies to a substantial part on the differences between the heterotic pools with respect to FT and NCLB resistance trait values (Figure 1). Our observation explains why we found neither for the whole set of genotypes (as we accounted for population structure) nor in the individual heterotic pools any overlap between SNPs associated with FT and NCLB (Table 2, 3, 4; Figure 3), and thus, no evidence of a pleiotropic effect of FT on NCLB resistance at the SNP level which is in accordance with results of [16].

Furthermore, we found no collocation between the SNPs associated with NCLB and $\operatorname{NCLB}_{F T}$ (Table 2; Figure 3 ) for the whole set of genotypes. This finding suggested that some of the SNP-NCLB associations outlined above for genes involved in heat and drought response might be due to an indirect link of these two traits with NCLB resistance as well as FT. Indeed, plants sensitive to drought stress have a tendency to show early senescense symptoms, which, in turn, leads to a higher sensitivity to necrotrophic pathogens such as Setosphaeria turcica [20].

Nevertheless, we identified SNPs in bins 7.04, 9.03, and 9.05 to be significantly associated with $\mathrm{NCLB}_{F T}$. The first SNP was located in GID1L2, a gibberellin receptor. Since gibberelin plays a role in basal disease resistance of various plant species [51,52], our finding might suggest that this gene is functionally involved in $\mathrm{NCLB}_{F T}$ resistance of maize.

The other two SNPs in bin 9.03 and 9.05 also significantly associated with $\mathrm{NCLB}_{F T}$ were located in genes with unknown function and a Sodium-Hydrogene exchanger, respectively, for which no obvious link to $\mathrm{NCLB}_{F T}$ is apparent. Nevertheless, we observed for both associations distinct $P$ value peaks supporting the hypothesis that these genes might be the causal genes or closely linked to them.

\section{Congruency of identified associations across heterotic pools}

For FT, we found in three of the four heterotic pools significantly associated SNPs in one (Flint) or two (Lancaster and Iodent) of the genes that where identified in the whole set of genotypes in the Vgt1 region (Table 2, 3; Additional file 1: Figure S1). In contrast, in the SSS pool, no significant association was detected for these loci. This is in accordance with earlier studies, in which QTLs were not detected in all examined populations in the Vgt1 region $[38,53,54]$. One reason could be that in the SSS pool no LD was present in the region between the causal gene and the examined polymorphisms. Another explanation might be that the early allele of $\mathrm{Vgt} 1$ does not occur in the SSS pool, because it flowers later than the other pools.

SNPs significantly associated with NCLB and NCLB $F T$ resistance were found in the SSS and Iodent pools, but not in the Flint and Lancaster ones (Table 3; Additional file 2: Figure S2 and Additional file 3: Figure S3). One explanation could be the difference in the extent of LD between the heterotic pools. The LD decays more rapidly in the Flint and Lancaster pools compared to the two other pools resulting in a lower genome coverage of 13 and $48 \%$ vs. 207 and $121 \%$. Furthermore, the number of markers required to detect associations explaining a significant part of the phenotypic variation (17 000 and 
65 000; respectively) in the Flint and Lancaster pools is higher than the number of SNPs actually available [29]. This could limit the power to detect associations for NCLB and $\mathrm{NCLB}_{F T}$ resistance in these two pools, whereas the number of required markers for the SSS and Iodent pools (4.000 and 7 000; respectively) is predicted to be sufficient.

In addition to the above described reasons for the imperfect congruency of the identified associations across heterotic pools are on one side sampling effects [55] but on the other side also epistatic interactions. Therefore, we searched for epistatic interactions between the significant SNPs identified in the whole set of genotypes and all the other markers. For FT and NCLB, highly significant epistatic interactions were detected (Figure 4) suggesting that epistasis contributes to the imperfect congruency of identified associations across different heterotic pools. This was even more important for NCLB, for which the epistatic interactions between markers explained as much genetic variation as their main effects (Additional file 4: Figure S4). These results are contradictory to the results of [16], who didn't find significant epistatic interactions between QTL markers and the others. The fact that elite breeding material was examined in our study, which has undergone a long process of selection, whereas the NAM population consists of multiple connected recombinant inbred line populations, could explain this difference.

\section{Relevance of the identified associations for practical breeding}

The significant SNP-FT associations identified in our study explained about $15 \%$ of the genetic variance (Table 2). This value is much lower than the value reported by [35]. This difference is due to the fact that they used (i) a stepwise forward regression, (ii) segregating populations, and (iii) a total of 5000 genotypes, which increase the power of QTL detection. In contrast to FT, the associations identified for NCLB resistance in our study explained only about $5 \%$ of the genetic variance (Table 2 ). This finding clearly suggests that the genetic architecture of NCLB has a higher genetic complexity than FT and, therefore, phenotypic but also marker-assisted selection will result in a lower gain of selection for the former than the latter. Nevertheless, for breeding applications, it seems more interesting to concentrate on $\mathrm{NCLB}_{F T}$ rather than NCLB, because the former is corrected for FT, the detected SNPs explain even a higher proportion of the genetic variance compared to the latter, and the correlation with population structure is lower for the former than the latter.

The proportion of the explained genetic variance was generally much higher in the individual pools than in the entire germplasm set (Table 2,3). Partly, this might be due to the reduced sample size leading to the overestimation of the allele effects and the explained genetic variance [56]. However, as the individual heterotic pools still comprise almost 400 genotypes, this overestimation is expected to be only small. More likely, our observation can be explained by different loci contributing to the variation of the examined traits in the individual heterotic pools (Table 3). Another explaination could also be the epistatic intereactions which importance differs among the heterotic pools. Finally, genome structure differences among the heterotic pools such as copy number or presence/absence variants [57] can explain our observation. Our finding suggests that despite association analysis across heterotic pools might be relevant for some traits to unravel the genetic architecture, marker-assisted selection within the individual heterotic pools, as praticed by plant breeders, is more promising than across heterotic pools.

Although we observed for FT highly significant epistatic interactions, these explained only a low proportion of the genetic variance compared to the main effects and, therefore, might be disregarded in marker-assisted selection for this trait. However, this was not true for NCLB as the epistatic interaction explained partly a higher proportion of the genetic variance than the main effects. Thus, taking epistasis into account for this trait should increase the efficiency of marker-assisted selection (Additional file 4: Figure S4).

\section{Conclusions}

We observed for FT, a trait for which already various genetic analyses in maize have been performed, a very well interpretable pattern of SNP associations, suggesting that data from practical plant breeding programs can be used to dissect polygenic traits. Furthermore, we described SNPs associated with NCLB and $\mathrm{NCLB}_{F T}$ resistance that are located in genes for which a direct link to the trait is discernable or which are located in bins of the maize genome for which previously QTLs have been reported. Some of the SNPs showed significant epistatic interactions with markers from the genetic background. The observation that the listed SNPs and their epistatic interactions explained in the entire germplasm set about $10 \%$ and in the individual heterotic pools up to $30 \%$ of the genetic variance suggest that significant progress towards improving the resistance of maize against NCLB by markerassisted selection is possible with these markers, without much compromising by a late flowering time. Furthermore, these regions are interesting for further research to understand the mechanisms of resistance to NCLB and diseases in general, because some of the genes identified were not annotated so far for these functions. However, as association mapping provides only statistical, i.e., indirect evidence for the function of the identified gene [58], a 
direct proof of the function of the identified alleles is still necessary.

\section{Methods}

\section{Plant materials, field experiments}

Our investigation was based on a set of 4149 maize inbred lines representing elite European and North American germplasm. The inbred lines are proprietary to the plant breeding company Limagrain (France) and were assigned by breeders to four heterotic pools, namely Flint, Lancaster, SSS, and Iodent.

In the years 2000 to 2009, these genotypes were evaluated for their per se performance in routine plant breeding trials, at different numbers of locations (2-7), with different experimental designs (randomised complete block design, nested design, etc.) and numbers of replicates (1-3). The experiments were either naturally infested or artificially infested with Setosphaeria turcica according to standard protocols [59]. All entries were evaluated for FT and NCLB resistance. FT was recorded in number of days to silking after June 1. NCLB was rated on a scale from 1 (sensitive) to 9 (resistant) at the level of individual plots.

\section{Molecular marker assays}

A subset of 1487 inbred lines randomly selected from the phenotyped inbreds regarding FT and NCLB were analyzed with 359 SSR and 8244 SNP markers (for details see [60]). The SSRs were selected over years by Limagrain with respect to their polymorphism information content value [61] in various sets of maize inbreds. The SNPs were discovered by sequencing 2973 amplicons in a development set of 30 diverse maize inbreds. From these, SNPs which showed an Illumina designability score $>0.4$ and were not in complete LD in the development set, were selected for genotyping the 1487 lines. The proportion of missing data was $5.1 \%$ for the SSRs and $2.7 \%$ for the SNPs. The amplicons had an average size of $477 \mathrm{bp}$ and contained on average three SNPs.

All markers were mapped in the IBM population [62]. Chromosomes 1 to 10 carried 59, 42, 41, 34, 36, 31, 36, 31, 27 , and 22 of the SSR markers, respectively. In addition, 1 $456,858,902,898,1002,633,578,632,699$, and 586 of the SNPs were mapped to chromosomes 1 to 10 , respectively. The total map length was $4265 \mathrm{cM}$ for the SSRs and 4378 cM for the SNPs. The physical positions of the markers were extract from Zea mays Genome Browser - Release 2.0.

Genotyping of the SSRs was performed by Limagrain Verneuil Holding (Riom, France) using standard protocols. Genotyping of the SNPs was performed by Biogemma (Clermont-Ferrand, France) using an Illumina Infinium iSelect chip.

\section{Statistical analyses}

\section{Phenotypic data analyses}

Phenotypic data were analysed based on the following mixed model:

$$
\begin{aligned}
y_{i j k l m}= & \mu+g_{i}+u_{j}+g_{i} * u_{j}+\theta_{j} t_{j k} \\
& +\beta_{j k} b_{j k l}+\rho_{j k l} r_{j k l m}+e_{j k l m},
\end{aligned}
$$

where $y_{i j k l m}$ is the phenotypic observation for the $i^{\text {th }}$ maize inbred line at the $j^{\text {th }}$ environment (year-location combination) in the $m^{\text {th }}$ replicate of the $l^{\text {th }}$ block in the $k^{\text {th }}$ trial, $\mu$ the intercept, $g_{i}$ the genetic effect of the $i^{t h}$ maize inbred line, $u_{j}$ the effect of the $j^{t h}$ environment, $g_{i} * u_{j}$ the genotype-by-environment interaction, $t_{j k}$ the effect of the $k^{\text {th }}$ trial in the $j^{\text {th }}$ environment, $b_{j k l}$ the effect of the $l^{\text {th }}$ block in the $k^{\text {th }}$ trial of the $j^{\text {th }}$ environment, $r_{j k l m}$ the effect of the $m^{\text {th }}$ replicate of the $l^{\text {th }}$ block in the $k^{\text {th }}$ trial of the $j^{\text {th }}$ environment, and $e_{j k l m}$ the residual. $\theta_{j}$ was a dummy covariate of value 1 in environments with several trials and of value 0 alternatively, $\beta_{j k}$ a dummy covariate of value 1 in environments with several trials and blocks and of value 0 alternatively, and $\rho_{j k l}$ a dummy covariate of value 1 in environments with several trials, blocks, and replicates and of value 0 alternatively.

Our study was based on data from 10 years and 23 locations spread over Europe, resulting in a total of 45 environments and, thus, the environmental factor was regarded as random. Error variances were assumed to be heterogeneous among environments. For calculating the adjusted entry mean $M_{i}$ for each of the 4149 inbred lines across all trials, we regarded $g_{i}$ as fixed and all other effects as random.

For estimation of variance components, except $\mu$, all effects including $g_{i}$ were regarded as random. Heritability on an entry mean basis was calculated for the phenotyped and genotyped inbred lines according to [63] for unbalanced breeding trials.

$\mathrm{NCLB}_{F T}$ was calculated according to [64]. A regression curve of NCLB against FT was computed (Figure 3). The vertical distance of an inbred's adjusted entry mean to the regression curve represented its $\mathrm{NCLB}_{F T}$ resistance value. Negative values indicated susceptible plants and positive values resistant plants.

Variance components were determined by the REML method. The mixed model analyses were performed with ASREML release 2.0 [65]. All other analyses were performed using the software R [66].

\section{Association analyses}

Single marker analysis: In the second step of our approach, we used the adjusted entry means for FT, NCLB, and $\mathrm{NCLB}_{F T}$ to test their associations with each of the 8244 SNP markers, using the QK method [67]: 


$$
M_{i p}=\mu+m_{p}+g_{i}^{*}+\sum_{u=1}^{z} D_{i u} v_{u}+\epsilon_{i p}
$$

where $M_{i p}$ is the adjusted entry mean of inbred $i$ carrying the $p^{t h}$ allele, $m_{p}$ is the effect of the $p^{\text {th }}$ allele of the SNP marker under consideration, $g_{i}^{*}$ the residual genetic effect of the $i^{t h}$ entry, $v_{u}$ the effect of the $u^{\text {th }}$ column of the population structure matrix $\mathbf{D}$, and $\epsilon_{i p}$ the residual. The variance-covariance matrix of the vector of random effects $g^{*}=\left\{g_{1}^{*}, \ldots, g_{1487}^{*}\right\}$ was assumed to be $\operatorname{Var}\left(g^{*}\right)=$ $2 \mathbf{K} \sigma_{g^{*}}^{2}$, where $\mathbf{K}$ was a $1487 \times 1487$ matrix of kinship coefficients that define the degree of genetic covariance between all pairs of inbreds, and $\sigma_{g^{*}}^{2}$ is the genetic variance estimated by REML. The variance-covariance matrix of the vector of errors $\epsilon_{i p}$ was assumed to be $\operatorname{Var}(\epsilon)=\mathbf{I} \sigma_{\epsilon}^{2}$.

The population structure matrix $\mathbf{Q}$ was calculated based on SSR markers using the software STRUCTURE [68] as described in detail by [60]. Per definition, the $z+1$ columns of the $\mathbf{Q}$ matrix add up to one. Thus, only the first $z$ columns were used as D matrix in our study, to achieve linear independence and, thus, avoid singularities. The kinship matrix K was calculated as described by [27] based on the SSR markers. In addition to the above described QK approach, we also examined other models: ANOVA, $\mathrm{Q}$, K and $\mathrm{K}_{T}$ (Additional file 6: Figure S6) for SNP markers but also for SSR markers [28]. In order to compare these different association mapping methods, expected $P$ values were calculated and the MSD between observed and expected $P$ values of all marker loci was then calculated as a measure of the deviation of the observed $P$ values from the uniform distribution [25].

Based on the Wald statistics, we performed a test for the presence of significant $(\alpha=0.05)$ SNP effects for each of the three traits. We dealt with the multiple testing problem by applying a Bonferroni and amplicon number based Bonferroni correction [31]. For the former, we used the total number of SNP markers to calculate the Bonferroni correction, whereas, for the latter, the correction was calculated using the number of amplicons from which the examined SNPs were derived. The proportion of the genetic variance explained by the significant SNPs was computed based on the relative reduction in genetic variance when the SNPs were added to the model [69]. Similarly, the proportion of genetic variance explained by the D matrix was calculated. Negative values were set to zero.

Heterotic pools: Similarly to the analyses conducted for the whole set of inbred lines, single marker analyses were conducted for each of the four heterotic pools. The same model was applied, except that no D matrix was considered in this case, as the population structure within the heterotic pools was modelled by the kinship matrix K.

Multiple forward regression: In order to take into account the LD between SNPs, we used in addition to the single marker analysis a multiple forward regression approach to identify, based on the above described QK model, those marker combinations which explain best the genotypic variation. A P-to-enter criterion was used. We added the SNP with the lowest $P$ value in the single marker analysis (if significant according to the amplicon based Bonferroni correction), as fixed cofactor in the analyses, when examining all remaining SNP markers for their association with the phenotype. For each of the three traits, this prodedure was repeated due to the high computational burden only two times and, thus a maximum of three SNPs could be selected.

Detection of epistasis: For each of the three traits, we performed a screen for epistatic interactions between the significant SNPs from the single marker analysis as well as multiple forward regression and all other SNP markers. The multiple testing problem was considered using the two different Bonferroni corrections.

The association analyses of SSR markers were performed with ASREML release 2.0 [65], whereas the association analyses of SNPs were performed with EMMA [70].

\section{Additional files}

Additional file 1: Figure S1. Genome-wide $P$ values for association analysis of flowering time within the different hererotic pools (Flint, Lancaster, SSS, lodent, respectively). The ten colors represent the ten chromosomes. The horizontal, doted and dashed-doted lines correspond to a nominal $5 \%$ significance threshold with Bonferroni and amplicon-wise Bonferroni correction, respectively. Significant $P$ values are represented by a star.

Additional file 2: Figure S2. Genome-wide $P$ values for association analysis of northern corn leaf blight resistance within the different hererotic pools (Flint, Lancaster, SSS, lodent, respectively). The ten colors represent the ten chromosomes. The horizontal, doted and dashed-doted lines correspond to a nominal 5\% significance threshold with Bonferroni and amplicon-wise Bonferroni correction, respectively. Significant $P$ values are represented by a star.

Additional file 3: Figure S3. Genome-wide $P$ values for association analysis of flowering time corrected northern corn leaf blight resistance within the different hererotic pools (Flint, Lancaster, SSS, lodent, respectively). The ten colors represent the ten chromosomes. The horizontal, doted and dashed-doted lines correspond to a nominal 5\% significance threshold with Bonferroni and amplicon-wise Bonferroni correction, respectively. Significant $P$ values are represented by a star.

Additional file 4: Figure S4. Significant epistatic interactions between the most significantly associated SNP for flowering time (FT) and northern corn leaf blight (NCLB) resistance and all other SNP markers for the entire set of 1487 maize inbred lines. $p_{G}$ is the proportion of the explained genotypic variance.

Additional file 5: Figure S5. $P$ values for association analysis of flowering time for the entire set of 1487 maize inbred lines on chromosome $\mathbf{8}$ in the $\mathbf{V g} \mathbf{t} \mathbf{~ r e g i o n}$. Significant $P$ values are represented by a star.

Additional file 6: Figure S6. Deviance of the QK mixed model association mapping method applied to northern corn leaf blight resistance of the entire germplasm set of the 1487 genotypes depending on threshold T. For details, see Materials and Methods. 


\section{Acknowledgements}

We are indebted to Limagrain Europe for providing the phenotyping and genotyping data; to S. Ducrocq, C. Lebreton, and Z. Karaman for inspiring discussions; and to A. Blanc and C. Boyard for their help extracting the data.

\section{Author details}

${ }^{1}$ Institute of Plant Breeding, Seed Science, and Population Genetics, University of Hohenheim, 70593 Stuttgart, Germany. ${ }^{2}$ Limagrain Europe, Bâtiment 1, CS 3911, 63720 Chappes, France. ${ }^{3}$ Current address: Limagrain GmbH, Breeding Station, Schönburg 6, 94060 Pocking, Germany. ${ }^{4}$ Max Planck Institute for Plant Breeding Research, Carl-von-Linne-Weg 10, 50829 Cologne, Germany.

\section{Author's contributions}

BS conceived the study. DVI performed all analyses and drafted the manuscript. BS, JPM, and AEM revised the manuscript. All authors read and approved the final version of the manuscript.

Received: 20 October 2011 Accepted: 30 April 2012

Published: 30 April 2012

\section{References}

1. Ullstrup AJ, Miles SR: The effects of some leaf blights of corn on grain yield. Phytopathology 1957, 47:331-336.

2. Perkins JM, Pedersen WL: Disease development and yield losses associated with northern corn leaf blight on corn. Plant Dis 1987 71:940-943

3. Fajemisin JM, Hooker AL: Predisposition to Diplodia-zeae stalk rot in corn affected by 3 Helminthosporium leaf blights. Phytopathology 1974, 64:1496-1499.

4. Hulbert SH, Webb CA, Smith SM, Sun Q: Resistance gene complexes: Evolution and utilization. Annu Rev Phytopathol 2001, 39:285-312.

5. Bentolila S, Guitton C, Bouvet N, Sailland A, Nykaza S, Freyssinet G: Identification of an RFLP marker tightly linked to the $\mathrm{Ht} 1$ gene in maize. Theor Appl Genet 1991, 82:393-398.

6. Ogliari JB, Guirnaraes MA, Aranha Carnargo LE: Chromosomal locations of the maize (Zea mays L.) HtP and $r t$ genes that confer resistance to Exserohilum turcicum. Genet Mol Biol 2007, 30:630-634.

7. Zaitlin D, DeMars S, Gupta M: Linkage of a second gene for NCLB resistance to molecular markers in maize. Maize Genet Coop NewsI 1992, 66:69-70.

8. Simcox KD, Bennetzen $\mathrm{JL}$ : Mapping the $H t N$ resistance gene to the long arm of chromosome 8. Maize Genet Coop News/ 1993, 67:118-119.

9. Hooker AL: Resistance to Helminthosporium turcicum from Tripsacum floridanum incorporated into corn. Maize Genet Coop News/1981, 55:87-88

10. Welz HG: Genetics and epidemiology of the pathosystem Zea mays/Setosphaeria turcica. Habilitation thesis, 1998, University of Hohenheim, Stuttgart.

11. Thakur RP, Leonard KJ, Leath S: Effects of temperature and light on virulence of Exserohilum turcicum on corn. Phytopathology 1989, 79:631-635.

12. Thakur RP, Leonard KJ, Jones RK: Characterization of a new race of Exserohilum turcicum virulent on corn with resistance gene $\mathrm{HtN}$. Plant Dis 1989, 73:151-155.

13. Windes JM, Pedersen WL: An isolate of Exserohilum turcicum virulent on maize inbreds with resistance gene $\mathrm{HtN}$. Plant Dis 1991, 75:430

14. Young ND: QTL mapping and quantitative disease resistance in plants. Annu Rev Phytopathol 1996, 34:479-501.

15. Wisser RJ, Balint-Kurti PJ, Nelson RJ: The genetic architecture of disease resistance in maize: A synthesis of published studies. Phytopathology 2006, 96:120-129

16. Poland JA, Bradbury PJ, Buckler ES, Nelson RJ: Genome-wide nested association mapping of quantitative resistance to northern leaf blight in maize. P Natl Acad Sci USA 2011, 108:6893-6898.

17. Ersoz ES, Yu J, Buckler ES: Applications of Linkage Disequilibrium and Association Mapping in Maize. Berlin: Springer Verlag; 2009: 173-195.

18. Abedon BG, Tracy WF: Corngrass 1 of maize (Zea mays L.) delays development of adult plant resistance to common rust (Puccinia sorghi Schw.) and European corn borer (Ostrinia nubilalis Hubner). $J$ Hered 1996, 87:219-223.
19. Century KS, Lagman RA, Adkisson M, Morlan J, Tobias R, Schwartz K, Smith A, Love J, Ronald PC, Whalen MC: Developmental control of Xa21-mediated disease resistance in rice. Plant J 1999, 20:231-236.

20. Rupe JC, Siegel MR, Hartman JR: Influence of environment and plant maturity on gray leaf spot of corn caused by Cercospora zeae-maydis. Phytopathology 1982, 72:1587-1591.

21. Jiang C, Edmeades GO, Armstead I, Lafitte HR, Hayward MD, Hoisington D: Genetic analysis of adaptation differences between highland and lowland tropical maize using molecular markers. Theor Appl Genet 1999, 99:1106-1119.

22. Schechert AW, Welz HG, Geiger HH: QTL for resistance to Setosphaeria turcica in tropical African maize. Crop Sci 1999, 39:514-523.

23. Stich B, Melchinger AE, Heckenberger M, Möhring J, Schechert A, Piepho $\mathrm{HP}$ : Association mapping in multiple segregating populations of sugar beet (Beta vulgaris L.). Theor Appl Genet 2008, 117:1167-1179.

24. Cullis B, Gogel B, Verbyla A, Thompson R: Spatial analysis of multi-environment early generation variety trials. Biometrics 1998 54:1-18.

25. Stich B, Möhring J, Piepho HP, Heckenberger M, Buckler ES, Melchinger AE: Comparison of mixed-model approaches for association mapping. Genetics 2008, 178:1745-1754.

26. Yu J, Pressoir G, Briggs WH, Vroh Bi I, Yamasaki M, Doebley JF, McMullen MD, Gaut BS, Nielsen DM, Holland JB, Kresovich S, Buckler ES: A unified mixed-model method for association mapping that accounts for multiple levels of relatedness. Nat Genet 2006, 38:203-208.

27. Zhao K, Aranzana MJ, Kim S, Lister C, Shindo C, Tang C, Toomajian C, Zheng H, Dean C, Marjoram P, Nordborg M: An Arabidopsis example of association mapping in structured samples. PLoS Genet 2007, 3:71-82.

28. Stich B, Melchinger AE: Comparison of mixed-model approaches for association mapping in rapeseed, potato, sugar beet, maize, and Arabidopsis. BMC Genomics 2009, 10.

29. Van Inghelandt D, Reif JC, Dhillon BS, Flament P, Melchinger AE: Extent and genome-wide distribution of linkage disequilibrium in commercial maize germplasm. Theor App/ Genet 2011, 123:11-20.

30. Cheverud JM: A simple correction for multiple comparisons in interval mapping genome scans. Heredity 2001, 87:52-58.

31. Lander ES, Botstein D: Mapping mendelian factors underlying quantitative traits using RFLP linkage maps. Genetics 1989 121:185-199.

32. Benjamini $Y$, Hochberg $Y$ : Controlling the false discovery rate: a practical and powerful approach to multiple testing. $J R$ Stastistical Soc 1995, 57:289-300

33. Urbany C, Stich B, Schmidt L, Simon L, Berding H, Junghans H, Niehoff KH, Braun A, Tacke E, Hofferbert HR, Luebeck J, Strahwald J, Gebhardt C: Association genetics in Solanum tuberosum provides new insights into potato tuber bruising and enzymatic tissue discoloration. $B M C$ Genomics 2011, 12:7.

34. Chardon F, Virlon B, Moreau L, Falque M, Joets J, Decousset L, Murigneux $A$, Charcosset A: Genetic architecture of flowering time in maize as inferred from quantitative trait loci meta-analysis and synteny conservation with the rice genome. Genetics 2004, 168:2169-2185.

35. Buckler ES, Holland JB, Bradbury PJ, Acharya CB, Brown PJ, Browne C, Ersoz E, Flint-Garcia S, Garcia A, Glaubitz JC, Goodman MM, Harjes C, Guil K, Kroon DE, Larsson S, Lepak NK, Li H, Mitchell SE, Pressoir G, Peiffer JA, Rosas MO, Rocheford TR, Romay MC, Romero S, Salvo S, Sanchez Villeda H, da Silva HS, Sun Q, Tian F, Upadyayula N, Ware D, Yates H, Yu J, Zhang Z, Kresovich S, McMullen MD: The genetic architecture of maize flowering time. Science 2009, 325:714-718.

36. Salvi S, Sponza G, Morgante M, Tomes D, Niu X, Fengler KA, Meeley R, Ananiev EV, Svitashev S, Bruggemann E, Li B, Hainey CF, Radovic S, Zaina G, Rafalski JA, Tingey SV, Miao GH, Phillips RL, Tuberosa R: Conserved noncoding genomic sequences associated with a flowering-time quantitative trait locus in maize. P Natl Acad Sci USA 2007, 104:11376-11381

37. Vladutu C, McLaughlin J, Phillips RL: Fine mapping and characterization of linked quantitative trait loci involved in the transition of the maize apical meristem from vegetative to generative structures. Genetics 1999, 153:993-1007.

38. Ducroca S, Madur D, Veyrieras JB, Camus-Kulandaivelu L, Kloiber-Maitz M, Presterl T, Ouzunova M, Manicacci D, Charcosset A: Key impact of Vgt1 
on flowering time adaptation in maize: Evidence from association mapping and ecogeographical information. Genetics 2008, 178:2433-2437.

39. Thornsberry JM, Goodman MM, Doebley J, Kresovich S, Nielsen D, Buckler ES: Dwarf8 polymorphisms associate with variation in flowering time. Nat Genet 2001, 28:286-289.

40. Andersen JR, Schrag T, Melchinger AE, Zein I, Lübberstedt T: Validation of Dwarf8 polymorphisms associated with flowering time in elite European inbred lines of maize (Zea mays L.). Theor Appl Genet 2005, 111:206-217.

41. Wang ZY, Kenigsbuch D, Sun L, Harel E, Ong MS, Tobin EM: A Myb-related transcription factor is involved in the phytochrome regulation of an Arabidopsis LHCB gene. Plant Cell 1997, 9:491-507.

42. Schaffer R, Ramsay N, Samach A, Corden S, Putterill J, Carre IA, Coupland $\mathrm{G}$ : The late elongated hypocotyl mutation of Arabidopsis disrupts circadian rhythms and the photoperiodic control of flowering. Cell 1998, 93:1219-1229.

43. Beló A, Zheng P, Luck S, Shen B, Meyer DJ, Li B, Tingey S, Rafalski A: Whole genome scan detects an allelic variant of fad 2 associated with increased oleic acid levels in maize. Mol Genet Genomics 2008, 279:1-10.

44. Balint-Kurti PJ, Yang J, Van Esbroeck G, Jung J, Smith ME: Use of a maize advanced intercross line for mapping of QTL for northern leaf blight resistance and multiple disease resistance. Crop Sci 2010, 50:458-466.

45. Welz HG, Geiger HH: Genes for resistance to northern corn leaf blight in diverse maize populations. Plant Breeding 2000, 119:1-14.

46. Wisser RJ, Kolkman JM, Patzoldt ME, Holland JB, Yu J, Krakowsky M, J NR, Balint-Kurti PJ: Multivariate analysis of maize disease resistances suggests a pleiotropic genetic basis and implicates a GST gene. $P$ Natl Acad Sci USA 2011, 108:7339-7344.

47. Kraakman ATW, Niks RE, Van den Berg PMMM, Stam P, van Eeuwijk FA: Linkage disequilibrium mapping of yield and yield stability in modern spring barley cultivars. Genetics 2004, 168:435-446.

48. Russell DA, Sachs MM: Protein synthesis in maize during anaerobic and heat stress. Plant Physiol 1992, 99:615-620.

49. Kizis D, Pages M: Maize DRE-binding proteins DBF1 and DBF2 are involved in rab17 regulation through the drought-responsive element in an ABA-dependent pathway. Plant J 2002, 30:679-689.

50. Saleh A, Lumbreras V, Lopez C, Dominguez-Puigjaner E, Kizis D, Pages Mm: Maize DBF1-interactor protein 1 containing an R3H domain is a potential regulator of DBF1 activity in stress responses. Plant J 2006 , 46:747-757.

51. Yang DL, Li Q, Deng YW, Lou YG, Wang MY, Zhou GX, Zhang YY, He ZH: Altered disease development in the eui mutants and Eui overexpressors indicates that gibberellins negatively regulate rice basal disease resistance. Mol Plant 2008, 1:528-537.

52. Jones JDG, Dangl JL: The plant immune system. Nature 2006, 444:323-329.

53. Austin DF, Lee M: Genetic resolution and verification of quantitative trait loci for flowering and plant height with recombinant inbred lines of maize. Genome 1996, 39:957-968.

54. Bouchez Ab, Hospital F, Causse M, Gallais A, Charcosset A: Marker-assisted introgression of favorable alleles at quantitative trait loci between maize elite lines. Genetics 2002, 162:1945-1959.

55. Melchinger AE, Utz HF, Schön CC: Quantitative trait locus (QTL) mapping using different testers and independent population samples in maize reveals low power of QTL detection and large bias in estimates of QTL effects. Genetics 1998, 149:383-403.

56. Beavis WD: QTL analyses: Power, precision, and accuracy. Boca Raton/New York: CRC Press, Inc.; 1998: 145-162.

57. Springer NM, Ying K, Fu Y, Ji T, Yeh CT, Jia Y, Wu W, Richmond T, Kitzman J, Rosenbaum H, Iniguez AL, Barbazuk WB, Jeddeloh JA, Nettleton D, Schnable PS: Maize inbreds exhibit high levels of copy number variation (CNV) and presence/absence variation (PAV) in genome content. PLoS Genet 2009, 5:11.

58. Andersen $J R$, Lübberstedt $\mathrm{T}$ : Functional markers in plants. Trends Plant Sci 2003, 8:554-560

59. Hooker AL: Relative efficiency of various methods in inducing field infections with Helminthosporium turcicum and Puccinia sorghi. Plant Dis Rep 1954, 38:173-177.
60. Van Inghelandt D, Melchinger AE, Lebreton C, Stich B: Population structure and genetic diversity in a commercial maize breeding program assessed with SSR and SNP markers. Theor App/ Genet 2010, 120:1289-1299.

61. Botstein D, White RL, Skolnick M, Davis RW: Construction of a genetic linkage map in man using restriction fragment length polymorphisms. Am J Hum Genet 1980, 32:314-331.

62. Lee M, Sharopova N, Beavis WD, Grant D, Katt M, Blair D, Hallauer A: Expanding the genetic map of maize with the intermated B73 X Mo17 (IBM) population. Plant Mol Biol 2002, 48:453-461.

63. Holland JB, Nyquist WE, Cervantes-Martinez CT: Estimating and interpreting heritability for plant breeding: An update. Plant Breed Rev 2003, 22:9-112.

64. Bormann C, Rickert A, Ruiz R, Paal J, Lubeck J, Strahwald J, Buhr K, Gebhardt C: Tagging quantitative trait loci for maturity-corrected late blight resistance in tetraploid potato with PCR-based candidate gene markers. Mol Plant-Microbe Interact 2004, 17:1126-1138.

65. Gilmour A, Gogel B, Cullis B, Thompson R: ASRem/ user guide release 2.0. Hermel Hempstead, UK: VSN International Ltd; 2006.

66. R Development Core Team: $R$ : A language and environment for statistical computing. Vienna, Austria: R Foundation for Statistical Computing; 2009.

67. Yu J, Buckler ES: Genetic association mapping and genome organization of maize. Curr Opin Biotechnol 2006, 17:155-160

68. Pritchard JK, Stephens M, Donnelly P: Inference of population structure using multilocus genotype data. Genetics 2000, 155:945-959.

69. Haseneyer G, Stracke S, Piepho HP, Sauer S, Geiger HH, Graner A: DNA polymorphisms and haplotype patterns of transcription factors involved in barley endosperm development are associated with key agronomic traits. BMC Plant Biol 2010, 10:5.

70. Kang HM, Zaitlen NA, Wade CM, Kirby A, Heckerman D, Daly MJ, Eskin E: Efficient control of population structure in model organism association mapping. Genetics 2008, 178:1709-1723.

doi:10.1186/1471-2229-12-56

Cite this article as: Van Inghelandt et al:: Genome-wide association mapping of flowering time and northern corn leaf blight (Setosphaeria turcica) resistance in a vast commercial maize germplasm set. BMC Plant Biology 2012 12:56.

\section{Submit your next manuscript to BioMed Central and take full advantage of:}

- Convenient online submission

- Thorough peer review

- No space constraints or color figure charges

- Immediate publication on acceptance

- Inclusion in PubMed, CAS, Scopus and Google Scholar

- Research which is freely available for redistribution 